\title{
Oral health of patients with intellectual disabilities
}

\author{
Abstracted from \\ Anders PL, Davis EL. \\ Oral health of patients with intellectual disabilities: A systematic review. \\ Spec Care Dentist 2010; 30: 110-117. \\ Address for correspondence: P. Landers, Department of Oral Diagnostic Sciences, \\ State University of New York at Buffalo School of Dental Medicine, \\ Buffalo, New York, USA. E-mail: planders@buffalo.edu
}

\section{Question:}

Is there a difference between the oral health of adults with intellectual disabilities (ID) and the general population?

Data sources Medline, PubMed, Clinical Queries and PsychLIT. Study selection The studies were evaluated independently by two reviewers and only included studies that had been published in English since 1975. The studies included had to meet three criteria; adult humans with mental retardation or a similar ID, had at least one quantitative measure of oral health status and the study compared the subjects to a control or comparison group without ID. Studies were assessed on the strength of evidence in three domains: quality, quantity and consistency. Data extraction and synthesis Owing to the heterogeneity of the data, meta-analyses were not attempted. The results were descriptive with no odds ratios or confidence intervals mentioned and gave an overview of oral health and ID.

Results 27 studies were included in the review. Studies were of variable quality with many being of a small sample size. People with ID were found to have poor oral hygiene and higher prevalence and greater severity of periodontal disease. Caries rates in people with ID are the same as or lower than the general population. The rates of untreated caries are consistently higher in people with ID.

Conclusions This review supports the need to develop strategies to increase patient acceptance for routine care, for additional training for dentists to provide care and for the development of more effective preventive strategies to minimise the need for this care.

\section{Commentary}

This study sets out to review the literature on 'the oral health of adults with intellectual disabilities' and would have benefitted from defining the term 'intellectual disabilities'. The research was carried out in the USA and it is important to appreciate the different terminology used in different countries, combined with the variety and complexity of disabilities limiting comparisons outside the host country.

How good is the systematic review? First it has to be asked whether the review was focussed enough to be a systematic review as the question asked was very broad. Secondly, can the results be interpreted sufficiently to draw evidence-based conclusions? There was no attempt to carry out meta-analyses on specific outcomes (eg, DMFT), some studies had small population numbers and there were substantial differences between the included studies, limiting the potential to generalise conclusions.

Papers from across the world were included in the review but the study could have benefitted from using wider search terms to capture more literature as well as the use of grey literature and additional contacts with experts in the field. The research question concentrated on intellectual disabilities without considering the implications of the consequences of confounding factors (educational ability or deprivation), or the severity of the disability which impact on oral health.

The time period (1975-2008) used to gather the literature has seen changes in the demographics of the population; ${ }^{1}$ with an increased life expectancy and increase in the number of patients with complex and long-term medical needs. There has also been a change in cultural values with tooth loss being less acceptable. ${ }^{2}$ There has been a shift away from living in institutions to living independently in the community with an appropriate level of care. As life expectancy has increased there has been a change in the patterns of oral disease and therefore the treatment plans associated with the disease and the disability.

A non-weighted scoring system, totalling 10, was used to assess the quality of the studies included in the review with a minimum score of 3 allowing inclusion into the study. Use of these types of scoring systems is not recommended by the Cochrane Collaboration. ${ }^{3}$

Owing to the methodological limitations the results of the review should be interpreted with care. The use of English only studies and narrow search terms must raise questions in relation to the reviews' conclusions which concentrate focus more on professionally focussed strategies to address disparities for patients with ID which have not yet been fully elucidated.

Emma O'Keefe

Department of Public Health, NHS Fife, Leven, Scotland, UK

\section{Practice point}

- Preventive programmes for these groups and their carers are important, as they are for other sectors of the population. They need to be delivered by people who understand the needs of people with learning disabilities.

1. Joint Advisory Committee for Special Care Dentistry. A case for need: proposal for a specialty in special care dentistry. London: JASCD, 2003.

2. Lewis D, Fiske J, Dougall A. Access to special care dentistry, part 7. Special care dentistry services: seamless care for people in their middle years- part 1 . Br Dent J 2008; 205: 305-317.

3. Higgins JPT, Green S (editors). Cochrane Handbook for Systematic Reviews of Interventions Version 5.0.2 [updated September 2009]. The Cochrane Collaboration, 2009. Available from www.cochrane-handbook.org.

Evidence-Based Dentistry (2010) 11, 81. doi:10.1038/sj.ebd.6400739 